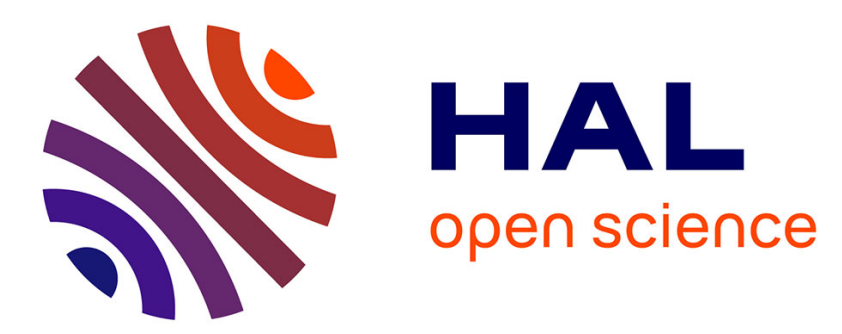

\title{
Review de: Ambroise de Milan, La fuite de siècle, texte critique, traduction et notes par Camille Gerzaguet (Sources chrétiennes, 576)
}

\author{
Marie Pauliat
}

\section{- To cite this version:}

Marie Pauliat. Review de: Ambroise de Milan, La fuite de siècle, texte critique, traduction et notes par Camille Gerzaguet (Sources chrétiennes, 576). Augustinianum : periodicum quadrimestre Collegii internationalis Augustiniani, 2016, 56, pp.286-290. 10.5840/agstm201656117 . halshs-03168438

\section{HAL Id: halshs-03168438 \\ https://shs.hal.science/halshs-03168438}

Submitted on 13 Mar 2021

HAL is a multi-disciplinary open access archive for the deposit and dissemination of scientific research documents, whether they are published or not. The documents may come from teaching and research institutions in France or abroad, or from public or private research centers.
L'archive ouverte pluridisciplinaire HAL, est destinée au dépôt et à la diffusion de documents scientifiques de niveau recherche, publiés ou non, émanant des établissements d'enseignement et de recherche français ou étrangers, des laboratoires publics ou privés. 
Fruit d'une thèse de doctorat soutenue par Camille Gerzaguet en novembre 2012, le volume 576 des «Sources chrétiennes » vient très heureusement compléter la série des œuvres d'Ambroise de Milan déjà publiées dans la collection. L'intérêt et la qualité de la publication ne font aucun doute, et ce sur trois plans complémentaires : cette nouvelle édition critique du De fuga saeculi repose sur la collation de nouveaux témoins manuscrits et sur l'établissement du premier stemma codicorum; le texte ainsi édité est assorti d'une traduction originale en langue française ; une introduction substantielle et d'abondantes notes guident la lecture du traité avec autant de précision que de pédagogie.

D'après le chapitre 5 de l'Introduction, le De fuga saeculi a été transmis à l'intérieur d'une tétralogie composée du De Isaac et anima, du De bono mortis, du De fuga saeculi et De Iacob et uita beata. Une édition à nouveaux frais de ce traité s'imposait: la précédente, établie par Charles Schenkl pour le CSEL 32.2 en 1897, ne reposait sur aucun stemma, de sorte qu'elle prenait en compte uniquement les variantes de la famille française de manuscrits. Sur les quatre-vingt-six manuscrits du De fuga, les trente plus anciens, antérieurs au milieu du $\mathrm{XII}^{\mathrm{e}}$ siècle, ont été collationnés pour la présente édition; leurs valeurs respectives sont évaluées à l'aide d'un stemma (pour l'intégralité de la discussion stemmatique, se reporter à C. Gerzaguet, Le "De fuga saeculi" d'Ambroise de Milan: transmission, diffusion et circulation de la tradition manuscrite (IXe-XIIe), dans Segno e Testo 12 (2014), 83-147). L'apport principal de cette édition réside dans l'utilisation conjointe des deux familles de manuscrits française et germano-italienne, ainsi que dans l'identification d'un nouveau témoin important, $L$ (le plus ancien témoin copié à Milan, où la mémoire textuelle de l'œuvre ambrosienne a été conservée avec la plus grande rigueur). Des «notes critiques » (p. 305-331) résolvent les plus problèmes de critique textuelle les plus importants. L'apparat critique qui en découle est d'une grande clarté. Il est complété par un apparat des sources et des citations bibliques.

Le reste de l'introduction apporte les éléments nécessaires à la compréhension de l'œuvre et de ses enjeux. Un premier chapitre s'attache à en définir la structure, en apparence décousue, comme celle de la plupart des textes ambrosiens. À la suite de Gérard Nauroy, Camille Gerzaguet rappelle que l'attention aux mots-crochets et aux procédés rhétoriques est nécessaire pour déterminer la composition du De fuga, composition qu'elle qualifie de «symphonique », délimitant sept «mouvements» (p. 19). Après un exorde en forme de captatio beneuolentiae, destiné à annoncer le thème du traité, cinq mouvements s'enchaînent : une exégèse allégorique des «villes-refuges» $(\mathrm{Nb} 35)$, librement inspirée de Philon d'Alexandrie dont la pensée est recomposée et enrichie de thèmes pauliniens pour servir le dessein propre d'Ambroise ; un commentaire des deux fuites de Jacob, dans une parénèse d'inspiration platonicienne ; une étude sur David, figure de l'âme du chrétien et de l'envol vers Dieu qu'elle est appelée à accomplir ; une analyse de l'opposition entre le bien d'en-haut, d'origine divine, et la malice d'ici-bas, diabolique ; la démonstration de la supériorité de la sagesse chrétienne sur la philosophie (celle-ci, en vertu du lieu commun du «larcin des philosophes », aurait emprunté à l'Écriture le thème de la fuite du monde). Enfin, la péroraison, construite en miroir par rapport à l'exorde, liste quatre exempla sur la fuite du monde.

Un deuxième chapitre revient sur les auditeurs de la prédication d'Ambroise, la publication de ses sermons et les lecteurs des œuvres ainsi recomposées. En effet, la complexité de la structure et des thèmes du De fuga s'accorde mal avec l'exigence d'une langue simple et sans artifice préconisée par Ambroise pour les sermons : l'écart entre la version orale et la forme publiée invite à postuler des destinataires différents pour l'une et l'autre versions. Le De fuga est vraisemblablement le fruit de la réélaboration de plusieurs 
prédications différentes, destinées à des publics variés (au point de vue à la fois de leur initiation chrétienne, de leur profil socio-économique et de leur degré d'instruction). En revanche, le lectorat visé par la version écrite serait composé en priorité d'initiés, dont certains mêlaient probablement le néoplatonisme à leurs croyances chrétiennes.

Le troisième chapitre propose de dater l'œuvre des toutes dernières années de la vie d'Ambroise, 395-396, sur la base de trois arguments : le De fuga se référerait à un ouvrage de grammaire, les Exempla elocutionum d'Arusianus Messius, datant d'après 395 ; par cette œuvre, Ambroise justifierait son exil volontaire de la cour impériale (désignée par «aula regia », fug. saec. 4, 19) en 394, lors de l'usurpation d'Eugène ; enfin, le De Isaac auquel le De fuga est lié dans la tétralogie doit être daté de 396.

Le quatrième chapitre présente les composantes théologiques, spirituelles et littéraires de la notion de «fuite du monde » dans le De fuga. Les éléments constitutifs du thème empruntent surtout au platonisme (médioplatonisme et néoplatonisme); l'influence stoïcienne est négligeable. Ils puisent aussi largement dans la pensée allégorique de Philon. Cependant, les emprunts aux philosophes et à l'exégète juif (sur les questions des genres de vie, du rapport au monde, et de la séparation du corps et de l'âme) ont tous fait l'objet d'une réorientation à partir de la doctrine chrétienne. Celle-ci repose sur des procédés d'écriture étudiés avec précision: décomposition et reconstruction des phrases, imbrication d'expressions philosophiques et bibliques, extraction d'une notion philosophique de son champ sémantique initial pour lui conférer un sens chrétien.

De fait, Ambroise rattache fermement l'idée de fuite du siècle à l'Ancien et au Nouveau Testament, spécialement à travers les expressions Deum sequi et sequela Christi. L'exégèse allégorique des villes-refuges et des deux fuites de Jacob donne aussi un fondement biblique aux deux éléments constitutifs du mouvement de fuite (le refuge à atteindre, Dieu, et la fuite loin du danger que représente le siècle). La figure de David sert à illustrer le rôle décisif de la grâce comme auxilium dans le processus de fuite. Le modèle du sacerdos, inspiré de l'idéal des Lévites, souligne finalement la nécessité de rompre les liens familiaux pour se consacrer à Dieu seul.

Enfin, Ambroise replace sa préoccupation du perfectionnement spirituel du croyant dans le cadre de l'économie du salut et à l'intérieur d'une perspective eschatologique. La fuite intra-mondaine qu'il préconise permettra d'être épargné le jour du Jugement dernier. Dans l'entre-deux situé entre la mort du Christ et la parousie, c'est-à-dire entre le salut déjà acquis et la gloire à venir, elle invite à fuir un monde éphémère frappé au coin de la vanité en tendant sans cesse vers les réalités éternelles dont sa mort a donné le gage.

Une «note complémentaire » (p. 142-146) précise la postérité du De fuga: Augustin l'emploie comme argument d'autorité durant la controverse pélagienne ; Florus en cite des extraits dans sa Collectio ambrosienne ; en revanche, la littérature monastique médiévale ne le mentionne quasiment pas (le contemptus mundi qu'elle prône, c'est-à-dire la dénonciation de la vanité du créé, ne recouvre pas exactement la fuga saeculi, tension permanente pour dépasser les réalités matérielles en s'attachant à Dieu).

La traduction est rédigée dans un français clair et précis, à la fois élégant et d'une grande fidélité au texte ambrosien. Des notes en facilitent la lecture : elles précisent certains termes, tissent les liens nécessaires avec d'autres sections de l'œuvre ou éclairent certaines allusions, en particulier philosophiques.

Nous n'indiquons qu'en vue d'une prochaine édition quelques coquilles relevées dans le volume. Les pages de l'article "Nauroy, «L'Écriture »" signalées en notes renvoient 
tantôt à la première édition, indiquée en bibliographie (G. Nauroy, L'Écriture dans la pastorale d'Ambroise de Milan, dans Le monde latin et la Bible, éds. J. FonTAINE-C. PIETRI, Paris 1985, 371-408) : c'est le cas p. 60, n. 4 ; et tantôt à une réimpression de l'article dans un recueil (G. Nauroy, Ambroise de Milan. Écriture et esthétique d'une exégèse pastorale, Bern 2003, p. 245-300) : c'est le cas de la p. 61, n. 2 et 4 ; p. 83, n. 1. Dans la bibliographie, manque la référence à $\mathrm{K}$. Rahner, Le début d'une doctrine des cinq sens spirituels chez Origène, dans Revue d'ascétique et de mystique 13 (1932), 113-145 (cité p. 191, n. 4).

Les passages du De fuga et inuentione de Philon d'Alexandrie cités dans l'apparat des sources ont été regroupés en annexe. Enfin, trois indices facilitent notablement la consultation du volume : un index scripturaire, un index des auteurs anciens et un index des parallela ambrosiens.

En saluant, pour conclure, les multiples apports de ce volume sur les deux plans indissociables de l'histoire du texte et des idées, nous ne pouvons qu'espérer la parution prochaine d'autres œuvres ambrosiennes dans la collection des « Sources chrétiennes ». 